\title{
A General Model for Matroids and the Greedy Algorithm
}

\author{
Ulrich Faigle* \\ Mathematisches Institut/ZAIK \\ Universität zu Köln \\ D-50931 Köln, Germany \\ Satoru Fujishige ${ }^{\dagger}$ \\ Research Institute for Mathematical Sciences \\ Kyoto University \\ Kyoto 606-8502, Japan
}

18 April, 2007

\begin{abstract}
We present a general model for set systems to be independence families with respect to set families which determine classes of proper weight functions on a ground set. Within this model, matroids arise from a natural subclass and can be characterized by the optimality of the greedy algorithm. This model includes and extends many of the models for generalized matroid-type greedy algorithms proposed in the literature and, in particular, integral polymatroids. We discuss the relationship between these general matroids and classical matroids and provide a Dilworth embedding that allows us to represent matroids with underlying partial order structures within classical matroids. Whether a similar representation is possible for matroids on convex geometries is an open question.
\end{abstract}

*e-mail: faigledzpr.uni-koeln.de

$\dagger$ e-mail: fujishig@kurims.kyoto-u.ac.jp Research supported by a Grant-in-Aid for Scientific Research of the Ministry of Education, Culture, Sports, Science and Technology of Japan. 


\section{Introduction}

A fundamental feature of combinatorial optimization is the fact that the optimality of the greedy algorithm with respect to arbitrary linear functions on finite independence systems is equivalent to the so-called Steinitz augmentation property and hence to the system giving rise to a matroid ( $c f$. $[7,18,28])$. Yet, many generalized models for the greedy algorithm have been established and proved useful. Most notably among them are Edmonds' [6] integral polymatroids that apply Hoffman's [19] linear programming approach to matroids. The polymatroid model in turn was further extended by allowing certain partial orders on the ground set in question (see, e.g., $[12,13,15,21,23])$.

On the other hand, already Dunstan, Ingleton, and Welsh [4] had introduced so-called supermatroids as a more general model for matroids. Extending distributive supermatroids, the combinatorial geometries on partially ordered sets of [9] yield a matroid type model for finite semimodular lattices. They furthermore admit an extension of the model for the greedy algorithm, which includes the polymatroid greedy algorithm (cf. [8]). Recently, so-called cg-matroids [17] have received attention. They generalize distributive supermatroids in that they allow a convex geometry as the underlying structure, whose lattice of closed sets is not necessarily distributive. For so-called strict cg-matroids a greedy algorithm exists and generalizes the matroid greedy algorithm (cf. [25]).

All those matroid generalizations have in common that they admit restrictions on the ground set while focusing on certain subclasses of linear functions that are found to be feasible for optimization by the accompanying greedy algorithms. The purpose of the present note is to provide a very general model for matroid independence systems that allow greedy optimization relative to certain classes of linear functions.

The key to the notion of a "matroid" in our approach is the link between a family $\mathcal{H}$ of subsets that includes the level sets of the linear functions to be optimized and a family $\mathcal{F}$ of feasible solutions. We first introduce our independence model and then exhibit the role the matroids play in this model with respect to greedy optimization. The striking feature of optimization under submodular constraints is Monge's [22] observation that optimal solutions have the structure of chains (see also, e.g., $[6,12,15]$ ). We formalize this aspect in the notion of the base chain property of generalized matroids. The base chain property then yields a convenient framework for matroid duality, which we sketch in Section 4. We then discuss matroids that are 
defined with respect to closure and co-closure systems $\mathcal{H}$. Important classes of such matroids, which include matroids on convex geometries, turn out to admit descriptions in terms of their associated rank functions. We finally investigate the relationship between $\mathcal{H}$-matroids and classical matroids. We provide the construction of a Dilworth embedding for matroids on distributive lattices of closed sets, which allows us to reduce the theory of such $\mathcal{H}$ matroids to classical matroid theory (which for integral polymatroids was already pointed out in [11]).

Whether general matroids on convex geometries allow a similar embedding into classical matroids is an open problem.

\section{Independence Systems}

We assume throughout to be given a finite ground set $E$.

\subsection{Constructible Families and Rank Functions}

Let $\mathcal{F}$ be a non-empty family of subsets of $E . \mathcal{F}$ is called constructible if for all $F \in \mathcal{F}$

(C) either $F=\emptyset$ or $F \backslash e \in \mathcal{F}$ for some $e \in F$.

Note that $(C)$ implies $\emptyset \in \mathcal{F}$. For any $F \in \mathcal{F}$, we set

$$
\Gamma(F)=\{e \in E \backslash F \mid F \cup e \in \mathcal{F}\}
$$

and call $F$ a basis of $\mathcal{F}$ if $\Gamma(F)=\emptyset$. So the bases of $\mathcal{F}$ are exactly the (inclusion-wise) maximal members of $\mathcal{F}$. Denote by $\mathcal{B}=\mathcal{B}(\mathcal{F})$ the collection of bases.

$\mathcal{F}$ induces a basis rank function $\rho$ on the collection of subsets of $E$ via

$$
\rho(S)=\max _{B \in \mathcal{B}}|S \cap B|=\max _{F \in \mathcal{F}}|S \cap F| .
$$

Note that $\rho$ is normalized (i.e., $\rho(\emptyset)=0$ ), and enjoys the unit-increase property

(UI) $\quad \rho(S) \leq \rho(T) \leq \rho(S)+|T \backslash S|$ for all $S \subseteq T \subseteq E$.

The restriction of $\mathcal{F}$ to a subset $S \subseteq E$ is the family

$$
\mathcal{F}(S)=\{F \in \mathcal{F} \mid F \subseteq S\} .
$$


Clearly, every restriction of a constructible family is constructible. Note, however, that the basis rank function $\rho_{S}$ of $\mathcal{F}(S)$ may differ from $\rho$. In general, one has the dominance relation

$$
\rho_{S}(X) \leq \rho(X) \text { for all } X \subseteq E .
$$

\section{$2.2 \mathcal{H}$-Independence}

Let $\mathcal{H}$ be a family of subsets with $\emptyset, E \in \mathcal{H}$. Then the constructible family $\mathcal{F}$ is said to form an independence system relative to $\mathcal{H}$ (or an $\mathcal{H}$-independence system) if

(I) for all $H \in \mathcal{H}$, there exists some $F \in \mathcal{F}(H)$ such that $|F|=\rho(H)$. In other words, $\mathcal{F}$ is an $\mathcal{H}$-independence system if and only if

$$
\rho(H)=\rho_{H}(H) \quad \text { for all } H \in \mathcal{H} .
$$

If $\mathcal{H}=2^{E}$, we refer to a $2^{E}$-independence system $\mathcal{F}$ simply as an "independence system" (or simplicial complex). Independence systems $\mathcal{F}$ are characterized by the fact that $F \in \mathcal{F}$ implies $F^{\prime} \in \mathcal{F}$ for all subsets $F^{\prime} \subseteq F$.

\subsubsection{The Intersection Property}

We say the $\mathcal{F}$ has the intersection property with respect to $\mathcal{H}$ (or the $\mathcal{H}$ intersection property) if

(IP) $F \cap H \in \mathcal{F} \quad$ for all $F \in \mathcal{F}, H \in \mathcal{H}$.

It follows immediately from the definition, that (IP) implies (I), i.e., every constructible family with the $\mathcal{H}$-intersection property is in particular an $\mathcal{H}$-independence system. Note that every simplicial complex has the intersection property. We illustrate the concept with an independence system of integral vectors. For a positive integer $N$ we identify $N$ with the set $\{1, \ldots, N\}$ in the following.

Example 2.1 Let the integer-valued function $f$ be defined on the collection of subsets of the set $N$ and define

$$
\mathbb{P}(f)=\left\{\mathbf{x} \in \mathbb{N}^{N} \mid \mathbf{x}(S)=\sum_{i \in S} x_{i} \leq f(S), S \subseteq N\right\},
$$


where $\mathbf{x}=\left(x_{i} \mid i=1, \ldots, N\right)$. Assuming that $f$ is bounded by $m \in \mathbb{N}$, consider $N$ pairwise disjoint sets $K_{i}=\left\{e_{i 1}, \ldots, e_{i m}\right\}(i=1, \ldots, N)$ and set $E=K_{1} \cup \cdots \cup K_{N}$. Every $\mathbf{x} \in \mathbb{P}(f)$ determines a subset

$$
\operatorname{id}\left(x_{1}, \ldots, x_{N}\right)=\bigcup_{i=1}^{N}\left\{e_{i j} \in K_{i} \mid j \leq x_{i}\right\} \subseteq E .
$$

The system $\mathcal{D}=\left\{\operatorname{id}\left(x_{1}, \ldots, x_{N}\right) \mid x_{i} \in \mathbb{N}, x_{i} \leq m\right\}$ is closed under intersections and unions with corresponding vector operations:

$$
\begin{aligned}
\operatorname{id}(\mathbf{x}) \cup \operatorname{id}(\mathbf{y}) & \longleftrightarrow \mathbf{x} \vee \mathbf{y}=\left(\ldots, \max \left(x_{i}, y_{i}\right), \ldots\right) \\
\operatorname{id}(\mathbf{x}) \cap \operatorname{id}(\mathbf{y}) & \longleftrightarrow \mathbf{x} \wedge \mathbf{y}=\left(\ldots, \min \left(x_{i}, y_{i}\right), \ldots\right) \\
\operatorname{id}(\mathbf{x}) \subseteq \operatorname{id}(\mathbf{y}) & \longleftrightarrow \mathbf{x} \leq \mathbf{y} .
\end{aligned}
$$

The family $\mathcal{F}=\{\operatorname{id}(\mathbf{x}) \mid \mathbf{x} \in \mathbb{P}(f)\}$ has the intersection property with respect to $\mathcal{D}$ and gives rise to the (vector) rank function

$$
\rho(\mathbf{y})=\max \{\mathbf{x}(N) \mid \mathbf{x} \in \mathbb{P}(f), \mathbf{x} \leq \mathbf{y}\}=\max _{F \in \mathcal{F}}|F \cap \operatorname{id}(\mathbf{y})| .
$$

More generally, if $\mathcal{D}$ is the system of (order-)ideals of a partially ordered set (poset) $\mathcal{P}=(E, \leq)$, the intersection property yields generalized independence systems that have been studied in the context of (distributive) supermatroids (see, e.g., [1, 4, 10, 27]). The integral polymatroids of Edmonds [6] can be understood as special distributive supermatroids ( $c f$. [11] and Example 6.2 below).

Convex geometries (in the sense of [5]) generalize systems of ideals of posets. The so-called (strict) $\mathrm{cg}$-matroids of [17] yield, in particular, independence systems with the intersection property relative to convex geometries.

The combinatorial geometries introduced in [9] include distributive supermatroids but do not have the intersection property in general (see Example 5.2 below).

\subsection{2 $\mathcal{H}$-Matroids}

An $\mathcal{H}$-independence system $\mathcal{F}$ is called an $\mathcal{H}$-matroid if for all $H \in \mathcal{H}$,

(M) all bases $B$ of the restriction $\mathcal{F}(H)$ have the same cardinality $|B|=$ $\rho(H)$. 
In the case of an independence system $\mathcal{F}$ (i.e., $\mathcal{H}=2^{E}$ ), (M) yields a ("classical") matroid.

Remark. In the case $\mathcal{H}=2^{E}$, property (M) is the so-called Steinitz exchange (or augmentation) property.

We exhibit a general submodularity property of the rank function of an $\mathcal{H}$-matroid. For large classes of families $\mathcal{H}$, this property is also sufficient to prove a normalized unit-increasing rank function to be a matroid rank function (see Section 5).

Lemma 2.1 Let $\mathcal{M}=(E, \mathcal{F})$ be an $\mathcal{H}$-matroid with rank function $\rho$. Let furthermore $H_{1}, H_{2} \in \mathcal{H}$ be such that $H_{1} \subseteq H_{2}$. Then for all $G_{1}, G_{2} \in \mathcal{H}$, one has

(S) $\left.\begin{array}{l}H_{1} \subseteq G_{1} \cap G_{2} \\ H_{2} \subseteq G_{1} \cup G_{2}\end{array}\right\} \quad \Longrightarrow \quad \rho\left(H_{1}\right)+\rho\left(H_{2}\right) \leq \rho\left(G_{1}\right)+\rho\left(G_{2}\right)$.

Proof. Consider $B_{1} \in \mathcal{F}\left(H_{1}\right)$ with $\left|B_{1}\right|=\rho\left(H_{1}\right)$. Because of $H_{1} \subseteq$ $H_{2}$ and the equicardinality property $(\mathbf{M}), B_{1}$ is contained in some $B_{2} \in$ $\mathcal{F}\left(H_{2}\right)$ with $\left|B_{2}\right|=\rho\left(H_{2}\right)$. So we conclude

$$
\begin{aligned}
\rho\left(H_{1}\right)+\rho\left(H_{2}\right) & \left.=\mid G_{1} \cap G_{2}\right) \cap B_{1}|+|\left(G_{1} \cup G_{2}\right) \cap B_{2} \mid \\
& =\left|G_{1} \cap B_{1}\right|+\left|G_{2} \cap B_{2}\right| \\
& \leq \rho\left(G_{1}\right)+\rho\left(G_{2}\right) .
\end{aligned}
$$

Hence, if $H_{1}, H_{2} \in \mathcal{H}$ are such that $H_{1} \cap H_{2} \in \mathcal{H}$ and $H_{1} \cup H_{2} \in \mathcal{H}$, we obtain from (S) the usual submodularity inequality

$$
\rho\left(H_{1} \cap H_{2}\right)+\rho\left(H_{1} \cup H_{2}\right) \leq \rho\left(H_{1}\right)+\rho\left(H_{2}\right) .
$$

\section{Linear Optimization}

Let $w: E \rightarrow \mathbb{R}$ be an arbitrary weight function on the ground set $E$. The function $w$ extends to arbitrary subsets $X \subseteq E$ via

$$
w(X)=\sum_{e \in X} w(e)
$$

Given families $\mathcal{F}$ and $\mathcal{H}$ of subsets of $E$ such that $\mathcal{F}$ is an $\mathcal{H}$-independence system, we are interested in the optimization problem

$$
\max _{F \in \mathcal{F}} w(F) .
$$




\subsection{The Greedy Algorithm}

The greedy algorithm is the following simple-minded procedure to solve (1), where we use the notation

$$
\Gamma_{w}(F)=\{e \in E \backslash F \mid w(e)>0, F \cup e \in \mathcal{F}\} \quad(F \in \mathcal{F}) .
$$

- Initialize: $F \leftarrow \emptyset$;

- Iterate: While $\Gamma_{w}(F) \neq \emptyset$ :

Choose $e \in \Gamma_{w}(F)$ of maximal possible weight $w(e)$;

Update $F \leftarrow(F \cup e)$;

- Output $F$.

We say that $w$ is $\mathcal{H}$-feasible if $\mathcal{H}$ contains all level sets of $w$, i.e.,

$$
W(\alpha)=\{e \in E \mid w(e) \geq \alpha\} \in \mathcal{H} \quad \text { for all } \alpha \in \mathbb{R} .
$$

Theorem 3.1 $\mathcal{M}=(E, \mathcal{F})$ is an $\mathcal{H}$-matroid if and only if the greedy algorithm is guaranteed to produce an optimal solution for (1) whenever $w$ is $\mathcal{H}$-feasible.

Proof. To see that the equicardinality property (M) necessarily holds for all bases of any $H \in \mathcal{H}$ if the greedy algorithm is optimal, consider the weight function $w=\chi_{H}$, where $\chi_{H}: E \rightarrow\{0,1\}$ is the characteristic function of $H \in \mathcal{H}$ with

$$
\chi_{H}(e)=1 \Longleftrightarrow e \in H .
$$

Any basis $B$ of $\mathcal{F}(H)$ is in accordance with the greedy algorithm. So optimality implies $w(B)=|B|=\rho(H)$.

To prove sufficiency, assume that $w_{1}>w_{2}>\cdots>w_{k}$ are the distinct values of $w(e)(e \in E)$, and that $w(e)>0$ for some $e \in E$. Setting $W_{i}=W\left(w_{i}\right)$, we have

$$
w=\sum_{i=1}^{k} \lambda_{i} \chi_{W_{i}}
$$

where $\lambda_{k}=w_{k}$ and $\lambda_{i}=w_{i}-w_{i+1} \geq 0$ for $i=1, \ldots, k$. Let $w_{p}>0$ be the smallest among the strictly positive values of $w_{1}, \ldots, w_{k}$ and set

$$
w^{(p)}=\sum_{i=1}^{p} \lambda_{i}^{\prime} \chi_{W_{i}}
$$


where $\lambda_{i}^{\prime}=\lambda_{i}$ for $i=1, \ldots, p-1$ and $\lambda_{p}^{\prime}=w_{p}$. By definition, the greedy algorithm will select a basis $B_{1}$ of $W_{1}$ and then extend $B_{1}$ to a basis $B_{2} \supseteq B_{1}$ of $W_{2}$ etc. and eventually output the basis $B_{p}$ of $W_{p}$. Because $\mathcal{M}$ is an $\mathcal{H}$-matroid, we have

$$
w\left(B_{p}\right)=\lambda_{1}^{\prime} \rho\left(W_{i}\right)+\lambda_{2}^{\prime} \rho\left(W_{2}\right)+\cdots+\lambda_{p}^{\prime} \rho\left(W_{p}\right)=w^{(p)}\left(B_{p}\right) .
$$

Since no member of $\mathcal{F}$ contains more than $\rho\left(W_{i}\right)$ elements of $W_{i}$ for all $i=1, \ldots, p$, it is clear that the greedy solution $B_{p}$ optimizes $w^{(p)}$ over $\mathcal{F}$. Because $w \leq w^{(p)}$ holds in general, $B_{p}$ must be optimal for $w$ as well.

From the proof of Theorem 3.1 we can easily see the following ( $c f .[18])$.

Theorem 3.2 For an $\mathcal{H}$-matroid $(E, \mathcal{F})$ and an $\mathcal{H}$-feasible positive weight $w: E \rightarrow \mathbb{R}$, let $\hat{B}$ be an optimal solution (basis) of (1) given by the greedy algorithm, and let $B$ be any basis of the $\mathcal{H}$-matroid. Suppose that elements of $\hat{B}$ and $B$ are indexed as

$$
\hat{B}=\left\{\hat{e}_{1}, \ldots, \hat{e}_{k}\right\}, \quad B=\left\{e_{1}, \ldots, e_{k}\right\}
$$

such that

$$
w\left(\hat{e}_{1}\right) \geq \cdots \geq w\left(\hat{e}_{k}\right), \quad w\left(e_{1}\right) \geq \cdots \geq w\left(e_{k}\right) .
$$

Then we have $w\left(\hat{e}_{i}\right) \geq w\left(e_{i}\right)$ for all $i=1, \ldots, k$.

We illustrate $\mathcal{H}$-feasible functions.

Example 3.1 Choosing $\mathcal{H}=\mathcal{D}$ in Example 2.1, the $\mathcal{H}$-feasible weight functions $w: E \rightarrow \mathbb{R}$ induce the so-called separable discrete concave functions (see, e.g., [26]) on integral vectors via

$$
\bar{w}(\mathbf{x})=\sum_{e \in \operatorname{id}(\mathbf{x})} w(e) .
$$

Taking $\mathcal{H}=\left\{K_{S} \mid S \subseteq N\right\}$ (with $K_{S}=\bigcup_{i \in S} K_{i}$ ), w $: E \rightarrow \mathbb{R}$ is $\mathcal{H}$ feasible precisely when $w$ is constant on each $K_{i}$, i.e., when (2) induces the linear function $\bar{w}(\mathbf{x})=w^{T} \mathbf{x}$ on $\mathbb{N}^{N}$. 
Example 3.2 If $\mathcal{H}$ is the collection of ideals of an arbitrary poset $P=$ $\left(E, \leq_{P}\right)$, then $w: E \rightarrow \mathbb{R}$ is $\mathcal{H}$-feasible if and only if for all $x, y \in E$,

$$
x \leq_{P} y \quad \Longrightarrow \quad w(x) \geq w(y) .
$$

Thus Theorem 3.1 implies the validity of the greedy algorithm in [8]. If $P$ is the trivial order, every $w: E \rightarrow \mathbb{R}$ is feasible and Theorem 3.1 yields the characterization of classical matroid independence systems by the greedy algorithm [7, 18].

\subsection{The Base Chain Property}

We say that an (arbitrary) family $\mathcal{B}$ of subsets $B \subseteq E$ has the base chain property with respect to $\mathcal{H}$ if

(BC) for every chain $H_{1} \subset \cdots \subset H_{k}$ of subsets $H_{i} \in \mathcal{H}$, there exists some $B \in \mathcal{B}$ such that $\rho\left(H_{i}\right)=\left|H_{i} \cap B\right|$ holds for all $i=1, \ldots, k$.

Theorem 3.3 Let $\mathcal{F}$ be an arbitrary constructible family with collection $\mathcal{B}$ of bases and assume that the greedy algorithm is guaranteed to produce an optimal solution for (1) whenever $w$ is $\mathcal{H}$-feasible. Then $\mathcal{B}$ has the base chain property $(\mathrm{BC})$.

Proof. Let $\left\{H_{1} \subset \ldots \subset H_{k}\right\}$ be a chain of subsets $H_{i} \in \mathcal{H}$ and consider the $\mathcal{H}$-feasible weight function

$$
w=\sum_{i=1}^{k} \lambda_{i} \chi_{H_{i}} \quad \text { with } \lambda_{1}>0, \ldots, \lambda_{k}>0 .
$$

Assuming w.l.o.g. $H_{k}=E$, note that the (optimal) greedy greedy solution yields a basis $B \in \mathcal{B}$ which does not depend on the absolute size of the weight parameters $\lambda_{i}>0$. Hence the choice $\lambda_{j}=1$ and $\lambda_{i} \approx 0$ for $i \neq j$ shows

$$
\left|H_{j} \cap B\right|=\rho\left(H_{j}\right) \quad(j=1, \ldots, k) .
$$

Theorem 3.3 says that every $\mathcal{H}$-matroid has the base chain property. The greedy algorithm may be viewed as just a procedure to generate an appropriate basis $B \in \mathcal{F}\left(W_{p}\right)$ for the chain

$$
W_{1} \subset W_{2} \subset \cdots \subset W_{p} .
$$


Moreover, there is a certain converse to Theorem 3.3:

Any $B \in \mathcal{F}$ satisfying $(\mathrm{BC})$ with $H_{i}=W_{i}$ as in the proof of Theorem 3.1 is an optimal solution for problem (1) if the weight function $w$ is $\mathcal{H}$-feasible and non-negative.

Let us call the pair $\mathcal{G}=(E, \mathcal{F})$ an $\mathcal{H}$-greedoid if $\mathcal{F}$ is a constructible family with the equicardinality property $(\mathrm{M})$. So $\mathcal{H}=2^{E}$ yields exactly the greedoids of [20]. In the latter case, the base chain property implies that the system $\mathcal{B}$ of bases of $\mathcal{G}$ is, in fact, the system of bases of a matroid $\tilde{\mathcal{M}}=(E, \tilde{\mathcal{F}})$ where

$$
\tilde{\mathcal{F}}=\{B \cap H \mid B \in \mathcal{B}, H \in \mathcal{H}\} .
$$

In general, however, a base system $\mathcal{B}$ with the base chain property (BC) does not necessarily induce an $\mathcal{H}$-matroid via (3). Suppose, for example, that $\mathcal{H}$ is graded (or Jordan-Dedekind) in the sense that the length of every maximal chain in $\mathcal{H}$ equals $|E|-1$. Then

$$
\tilde{\mathcal{F}}=\{B \cap H \mid B \in \mathcal{B}, H \in \mathcal{H}\}
$$

is an $\mathcal{H}$-independence system, but not necessarily an $\mathcal{H}$-matroid. If $\mathcal{H}$ is not only Jordan-Dedekind but also closed under taking intersections, (BC) implies that $\tilde{\mathcal{M}}=(E, \tilde{\mathcal{F}})$ is an $\mathcal{H}$-matroid (see Example 5.1 in Section 5). This is a consequence of the following observation:

Corollary 3.1 Let $\mathcal{B}$ be a family of subsets of $E$ with the base chain property (BC) relative to $\mathcal{H}$. Then the associated rank function $\rho$ is submodular (in the sense of condition (S) of Lemma 2.1).

\section{Duality}

The discussion of the base chain property (BC) suggests a duality framework for $\mathcal{H}$-matroids. We assume to be given a (non-empty) system $\mathcal{B}$ of equicardinal subsets $B \subseteq E$, whose members are bases. As before, we define an associated rank function

$$
\rho(S)=\max _{B \in \mathcal{B}}|S \cap B|
$$


and consider a subset family $\mathcal{H}$ with $\emptyset, E \in \mathcal{H}$. Define the dual of an (arbitrary) family $\mathcal{T}$ of subsets of $E$ as the family

$$
\mathcal{T}^{*}=\{E \backslash T \mid T \in \mathcal{T}\} .
$$

Then the dual $\mathcal{B}^{*}$ of a base system $\mathcal{B}$ is again a base system. Clearly, $B \in \mathcal{B}$ maximizes the intersection with a set $S$ if and only if $B^{*}=E \backslash B$ maximizes the intersection with $S^{*}=E \backslash S$. Hence we can represent the rank function $\rho^{*}$ of $\mathcal{B}^{*}$ in terms of $\rho$ via

$$
\rho^{*}(S)=|S|-[\rho(E)-\rho(E \backslash S)] \quad \text { for all } S \subseteq E \text {. }
$$

Moreover, we note:

Proposition 4.1 The base system $\mathcal{B}$ satisfies $(\mathrm{BC})$ relative to $\mathcal{H}$ if and only if its dual $\mathcal{B}^{*}$ satisfies $(\mathrm{BC})$ relative to $\mathcal{H}^{*}$.

\section{Matroids on Closure Spaces}

Let $\mathcal{H}$ be as before a subset family with $\emptyset, E \in \mathcal{H}$. Recall that $\mathcal{H}$ is a closure system (and the pair $(E, \mathcal{H})$ a closure space) if $\mathcal{H}$ is intersection-closed, i.e.,

$$
H_{1} \cap H_{2} \in \mathcal{H} \quad \text { for all } H_{1}, H_{2} \in \mathcal{H} .
$$

Assume henceforth that $\mathcal{H}$ is a closure system and associate with $\mathcal{H}$ the closure operator

$$
X \mapsto \bar{X}=\bigcap\{H \in \mathcal{H} \mid X \subseteq H\} \quad(X \subseteq E) .
$$

$X$ is called closed if $X=\bar{X}$. So $\mathcal{H}$ is precisely the collection of closed sets. Moreover, $(\mathcal{H}, \subseteq)$ is a lattice with the infimum and supremum operations

$$
\begin{aligned}
& H_{1} \wedge H_{2}=H_{1} \cap H_{2} \\
& H_{1} \vee H_{2}=\overline{H_{1} \cup H_{2}} .
\end{aligned}
$$

We say that $H_{2}$ covers $H_{1}$ (denoted $H_{1} \prec H_{2}$ ) if $H_{1} \subset H_{2}$ holds and for all $H \in \mathcal{H}$,

$$
H_{1} \subset H \subseteq H_{2} \quad \Longrightarrow \quad H=H_{2} .
$$




\subsection{Matroids from Rank Functions}

Let $(E, \mathcal{H})$ be a closure space and consider the function $r: \mathcal{H} \rightarrow \mathbb{N}$ such that for all $H_{1}, H_{2} \in \mathcal{H}$,

( $\left.\mathrm{R}_{0}\right) r\left(H_{1}\right)=0 \quad$ if $H_{1}=\emptyset \quad$ (normalization);

(R $\left.\mathrm{R}_{1}\right) H_{1} \prec H_{2} \quad \Longrightarrow \quad r\left(H_{1}\right) \leq r\left(H_{2}\right) \leq r\left(H_{1}\right)+1 \quad$ (unit-increase);

$\left(\mathrm{R}_{2}\right) H_{1} \prec\left(H_{1} \vee H_{2}\right) \quad \Longrightarrow \quad r\left(H_{1} \wedge H_{2}\right)+r\left(H_{1} \vee H_{2}\right) \leq r\left(H_{1}\right)+r\left(H_{2}\right)$.

Associate with $r$ the family $\mathcal{F}=\mathcal{F}(r)$ of subsets of $E$ that can be obtained via the following algorithmic procedure:

- Initialize: Choose some $H \in \mathcal{H}$ and a maximal chain $\mathbf{M}_{H}$ from $\emptyset$ to $H$ :

$$
\mathbf{M}_{H}=\left\{\emptyset=M_{0} \prec M_{1} \prec \ldots \prec M_{k}=H\right\} ;
$$

- Choose a sequence $\pi=u_{1} \ldots u_{k}$ of representatives $u_{i} \in M_{i} \backslash M_{i-1}$,

- Output the set $F=\left\{u_{i} \mid r\left(M_{i}\right)=r\left(M_{i-1}\right)+1\right\}$.

It follows directly from the algorithmic definition that $\mathcal{F}$ is constructible. Moreover, in view of the unit increase property $\left(\mathrm{R}_{1}\right)$, each basis $B$ of the restriction $\mathcal{F}(H)$ has cardinality $|B|=r(H)$. So $\mathcal{F}$ has the equicardinality property (M) of a matroid.

Theorem 5.1 If $\left(\mathrm{R}_{0}\right)-\left(\mathrm{R}_{2}\right)$ hold for $r, \mathcal{F}$ is an $\mathcal{H}$-independence system and $\mathcal{M}=(E, \mathcal{F})$ an $\mathcal{H}$-matroid with rank function $r$.

Proof. It remains to verify that $r(H)$ equals the basis rank $\rho(H)$ of $H \in \mathcal{H}$ relative to $\mathcal{F}$. Arguing by induction on $|E|$, we may assume $H=E$ without loss of generality.

So let $B \in \mathcal{B}$ be an arbitrary independent set and assume that $B$ arises from the chain $\mathbf{M}=\left\{\emptyset=M_{0} \prec \ldots \prec M_{k}=E\right\}$ and the representatives $\pi=u_{1}, \ldots, u_{k}$ via

$$
B=\left\{u_{i} \mid r\left(M_{i}\right)=r\left(M_{i-1}\right)+1\right\} .
$$

Consider the function $f: \mathcal{H} \rightarrow \mathbb{R}$ where

$$
f(A)=r(A)-|A \cap B| .
$$

We claim $f(A) \geq 0$. One easily checks 
- $f\left(M_{i}\right)=0$ for $i=0,1, \ldots, k$;

- $H_{1} \prec\left(H_{1} \vee H_{2}\right) \Longrightarrow f\left(H_{1} \wedge H_{2}\right)+f\left(H_{1} \vee H_{2}\right) \leq f\left(H_{1}\right)+$ $f\left(H_{2}\right)$.

Suppose now that the claim is false and $A \in \mathcal{H}$ a counterexample of minimal cardinality. Let $j$ be the index such that $A \subseteq M_{j}$ and $A \nsubseteq M_{j-1}$ holds. Then we obtain

$$
0>f(A) \geq f\left(A \wedge M_{j-1}\right)+f\left(M_{j}\right)-f\left(M_{j-1}\right)=f\left(A \wedge M_{j-1}\right) \geq 0,
$$

which is a contradiction. So the claim is true and $|H \cap B| \leq r(H)$ follows for all $H \in \mathcal{H}$. Hence we conclude

$$
r(H) \leq \rho(H)=\max _{B \in \mathcal{F}}|H \cap B| \leq r(H) \quad \text { and thus } \quad r(H)=\rho(H) .
$$

Example 5.1 Let $\mathcal{C}=(E, \mathcal{H})$ be a convex geometry, i.e., a closure space such that for all $H_{1}, H_{2} \in \mathcal{H}$,

$$
H_{1} \prec H_{2} \quad \Longrightarrow \quad\left|H_{2}\right|=\left|H_{1}\right|+1 .
$$

(Note that convex geometries are Jordan-Dedekind.) Then we have

$$
H_{1} \prec\left(H_{1} \vee H_{2}\right) \quad \Longrightarrow \quad H_{1} \vee H_{2}=H_{1} \cup H_{2} .
$$

So Theorem 5.1 implies that the submodularity property $(S)$ of Lemma 2.1 is necessary and sufficient for a normalized unit-increasing function $r: \mathcal{H} \rightarrow$ $\mathbb{N}$ to be the rank function of an $\mathcal{H}$-matroid.

The $\mathcal{H}$-matroid $\mathcal{M}=(E, \mathcal{F})$ is a strict cg-matroid in the sense of [17] when $\mathcal{F} \subseteq \mathcal{H}$ holds.

Example 5.2 Let $P=(E, \leq)$ be a poset with collection $\mathcal{D}$ of ideals. Then $(E, \mathcal{D})$ is in particular a convex geometry. The $\mathcal{D}$-matroids are essentially the combinatorial geometries of [9] and the strict $\mathcal{D}$-matroids are the distributive supermatroids of [4].

Remark. We point out that independence systems of type $\mathcal{F}(r)$ do not have the intersection property in general. 


\subsection{Matroids on Co-Closure Spaces}

Let $\mathcal{C}=(E, \mathcal{H})$ be a closure space. Then the dual structure $\mathcal{C}^{*}=\left(E, \mathcal{H}^{*}\right)$ is a so-called co-closure space. $\mathcal{H}^{*}$ is union-closed and $\left(\mathcal{H}^{*}, \subseteq\right)$ is antiisomorphic to the lattice $(\mathcal{H}, \subseteq)$ under the supremum and infimum operations

$$
\begin{aligned}
S_{1} \vee^{*} S_{2} & =S_{1} \cup S_{2}, \\
S_{1} \wedge^{*} S_{2} & =\bigcup\left\{S \in \mathcal{H}^{*} \mid S \subseteq S_{1} \cap S_{2}\right\}
\end{aligned}
$$

Let $r: \mathcal{H}^{*} \rightarrow \mathbb{N}$ be normalized and unit-increasing and consider the property

$\left(\mathrm{R}_{2}^{*}\right)\left(S_{1} \wedge^{*} S_{2}\right) \prec S_{1} \quad \Longrightarrow \quad r\left(S_{1} \wedge^{*} S_{2}\right)+r\left(S_{1} \vee^{*} S_{2}\right) \leq r\left(S_{1}\right)+r\left(S_{2}\right)$.

As before, we associate with $r$ the family $\mathcal{F}=\mathcal{F}(r)$ of subsets of $E$ that can be obtained via the following algorithmic procedure:

- Initialize: Choose some $S \in \mathcal{H}^{*}$ and a maximal chain $\mathbf{M}_{S}$ from $\emptyset$ to $S$ :

$$
\mathbf{M}_{S}=\left\{\emptyset=M_{0} \prec M_{1} \prec \cdots \prec M_{k}=S\right\} ;
$$

- Choose a sequence $\pi=u_{1} \ldots u_{k}$ of representatives $u_{i} \in M_{i} \backslash M_{i-1}$,

- Output the set $F=\left\{u_{i} \mid r\left(M_{i}\right)=r\left(M_{i-1}\right)+1\right\}$.

Again, it is clear that the bases $B$ of $\mathcal{F}(S)$ share the same cardinality $|B|=r(S)$. In order to establish $(E, \mathcal{M})$ as an $\mathcal{H}^{*}$-matroid, we need an additional assumption on $\mathcal{H}^{*}$.

We call the co-closure system $\mathcal{H}^{*}$ locally modular if for all $S_{1}, S_{2} \in \mathcal{H}^{*}$, (LM)

$$
S_{1} \wedge^{*} S_{2} \prec S_{1} \quad \Longrightarrow \quad S_{1} \wedge^{*} S_{2}=S_{1} \cap S_{2} .
$$

Theorem 5.2 Assume that $\mathcal{H}^{*}$ is locally modular and $r: \mathcal{H}^{*} \rightarrow \mathbb{N}$ is a normalized unit-increasing function with property $\left(\mathrm{R}_{2}^{*}\right)$. Then $\mathcal{M}=(E, \mathcal{F}(r))$ is an $\mathcal{H}^{*}$-matroid with rank function $r$.

Proof. The key observation under the assumption of local modularity is the following. Given an arbitrary $B \in \mathcal{F}$,

$$
f(S)=r(S)-|S \cap B|
$$


yields a function on $\mathcal{H}^{*}$ with the property

$$
\left(S_{1} \wedge^{*} S_{2}\right) \prec S_{1} \quad \Longrightarrow \quad f\left(S_{1} \wedge^{*} S_{2}\right)+f\left(S_{1} \vee^{*} S_{2}\right) \leq f\left(S_{1}\right)+f\left(S_{2}\right) .
$$

One may now argue in analogy with the proof of Theorem 5.1 and choose, if possible, an $S \in \mathcal{H}^{*}$ of maximal cardinality such that $f(S)<0$. Letting $j$ be such that $S \supseteq M_{j-1}$ but $S \nsupseteq M_{j}$, one arrives at a contradiction as before.

\subsection{Duality for Convex Geometries and Antimatroids}

An antimatroid (cf. [20]) is the co-closure space $\mathcal{C}^{*}=\left(E, \mathcal{H}^{*}\right)$ associated with a convex geometry $\mathcal{C}=(E, \mathcal{H})$. Thus we have for all $S_{1}, S_{2} \in \mathcal{H}^{*}$

$$
S_{1} \prec S_{2} \quad \Longrightarrow \quad\left|S_{2}\right|=\left|S_{1}\right|+1
$$

and therefore conclude local modularity:

$$
S_{1} \wedge^{*} S_{2} \prec S_{1} \quad \Longrightarrow \quad S_{1} \wedge^{*} S_{2}=S_{1} \cap S_{2} .
$$

Let $r: \mathcal{H} \rightarrow \mathbb{N}$ be an arbitrary function and define $r^{*}: \mathcal{H}^{*} \rightarrow \mathbb{N}$ via

$$
r^{*}(S)=|S|-[r(E)-r(E \backslash S)] .
$$

It is straightforward to verify that $r$ is normalized and unit-increasing if and only if $r^{*}$ is normalized and unit-increasing. It follows that (5) establishes a one-to-one correspondence between the $\mathcal{H}$-matroid rank functions $r$ and the $\mathcal{H}^{*}$-matroid rank functions $r^{*}$ since

$$
r(H)=|H|-\left[r^{*}(E)-r^{*}(E \backslash H)\right] \quad(H \in \mathcal{H}) .
$$

Note that this duality framework for matroids on convex geometries and matroids on antimatroids is compatible with the duality framework for general base systems of Section 4 .

\section{Closures and the Dilworth Completion}

\subsection{Closures}

Let $\mathcal{C}=(E, \mathcal{H})$ be a closure space and $\mathcal{F}$ an $\mathcal{H}$-independence system with rank function $\rho$. For any $S \subseteq E$, we set

$$
\sigma(S)=\{e \in E \mid \rho(\overline{S \cup e})=\rho(\bar{S})\} .
$$


In the case when $\sigma(S)=S$, we refer to $S$ as $\rho$-closed. We want to know under what conditions $S \mapsto \sigma(S)$ is a closure operator. Since $E$ is clearly $\rho$-closed, we thus ask when the collection $\Sigma$ of $\rho$-closed sets is intersectionclosed.

Since $X \mapsto \bar{X}$ is a closure operator, we immediately obtain

$$
\overline{\sigma(S)}=\sigma(S) \in \mathcal{H} \quad \text { and thus } \quad \Sigma \subseteq \mathcal{H} .
$$

Hence it suffices to study the action of $\sigma$ on $\mathcal{H}$. To this end, observe for any $H \in \mathcal{H}$ the slightly more convenient representation

$$
\sigma(H)=\bigvee\{G \in \mathcal{H} \mid \rho(H \vee G)=\rho(H)\}
$$

Theorem 6.1 $S \mapsto \sigma(S)$ is a closure operator if $\rho$ is submodular on $\mathcal{H}$ in the sense

$$
\rho\left(H_{1} \wedge H_{2}\right)+\rho\left(H_{1} \vee H_{2}\right) \leq \rho\left(H_{1}\right)+\rho\left(H_{2}\right) \quad \text { for all } H_{1}, H_{2} \in \mathcal{H} .
$$

Conversely, provided $\mathcal{H}$ is also union-closed, $S \mapsto \sigma(S)$ is a closure operator only if $\rho$ is submodular on $\mathcal{H}$.

Proof. Let $\rho$ be submodular and $H_{1}, H_{2} \in \mathcal{H} \rho$-closed. Suppose that $D=H_{1} \cap H_{2}=H_{1} \wedge H_{2}$ is not $\rho$-closed. So there is some $G \in \mathcal{H}$ such that $\rho(D \vee G)=\rho(D)$. Assume w.l.o.g. $G \nsubseteq H_{1}$. Since $H_{1}=\sigma\left(H_{1}\right)$, we have $\rho\left(H_{1} \vee G\right)>\rho\left(H_{1}\right)$ and obtain the contradiction

$$
\rho\left(H_{1}\right)<\rho\left(H_{1} \vee G\right) \leq \rho\left(H_{1}\right)+\rho(D \vee G)-\rho\left(H_{1} \wedge(D \vee G)\right) \leq \rho\left(H_{1}\right) .
$$

Hence we find that the collection $\Sigma$ of $\rho$-closed sets is intersection-closed if $\rho$ is submodular.

Conversely, assume that $\mathcal{H}$ is union closed and $H \mapsto \sigma(H)$ is a closure operator. We prove the submodularity inequality by induction on the $\mid H_{1} \cup$ $H_{2} \mid$. Clearly, the inequality is true if $H_{1} \subseteq H_{2}$ or $H_{2} \subseteq H_{1}$. Therefore, we may assume w.l.o.g. that there exists some $H_{2}^{\prime} \prec H_{2}$ with $H_{2}^{\prime} \supseteq H_{1} \cap H_{2}$. By induction, we thus have

$$
\begin{aligned}
\rho\left(H_{1} \cap H_{2}\right)+\rho\left(H_{1} \cup H_{2}^{\prime}\right) & =\rho\left(H_{1} \cap H_{2}^{\prime}\right)+\rho\left(H_{1} \cup H_{2}^{\prime}\right) \\
& \leq \rho\left(H_{1}\right)+\rho\left(H_{2}^{\prime}\right) .
\end{aligned}
$$


If $\rho\left(H_{2}\right)=\rho\left(H_{2}^{\prime}\right)+1$, the inequality follows trivially in view of the unitincrease property of $\rho$. So we may assume $\rho\left(H_{2}^{\prime}\right)=\rho\left(H_{2}\right)$ for the remainder of the proof.

If $\rho\left(H_{1} \vee H_{2}^{\prime}\right)=\rho\left(H_{1} \vee H_{2}\right)$, there is nothing left to show. On the other hand, if $\rho\left(H_{1} \vee H_{2}^{\prime}\right)<\rho\left(H_{1} \vee H_{2}\right)$ is true, we have $H_{2} \nsubseteq \sigma\left(H_{1} \vee H_{2}^{\prime}\right)$ and therefore also $H_{2} \nsubseteq \sigma \sigma\left(H_{2}^{\prime}\right)$, which means $\rho\left(H_{2}\right)>\rho\left(H_{2}^{\prime}\right)$.

Example 6.1 Let $\mathcal{H}$ be intersection- and union-closed. Then Lemma 2.1 implies that the rank function of every $\mathcal{H}$-matroid is submodular. Hence Theorem 6.1 characterizes the rank functions that arise from $\mathcal{H}$-matroids. In particular, the family $\mathcal{D}$ of ideals of any poset $P=(E, \leq)$ is union- and intersection-closed. Consequently, we find that every $\mathcal{D}$-matroid is characterized by the fact that $S \mapsto \sigma(S)$ is a closure operator.

Remark. The converse part in Theorem 6.1 may be false on general convex geometries. Assume, for example, that $\mathcal{C}=(E, \mathcal{H})$ is a convex geometry that admits sets $H_{1}, H_{2} \in \mathcal{H}$ with $H_{1} \cup H_{2} \notin \mathcal{H}$. Then $r(H)=$ $|H|$ is normalized and unit-increasing, but not submodular on $\mathcal{H}$ (because $\left.\left|H_{1} \vee H_{2}\right|>\left|H_{1} \cup H_{2}\right|\right)$. Yet, the associated operator $S \mapsto \sigma(S)$ is a closure operator with collection $\Sigma=\mathcal{H}$ of closed sets.

\subsection{The Dilworth Completion}

Let $\mathcal{C}=(E, \mathcal{H})$ be a closure space and $\mathcal{M}=(E, \mathcal{F})$ an $\mathcal{H}$-matroid with rank function $\rho$. If $\rho$ is submodular on $\mathcal{H}$, the system $\Sigma$ of $\rho$-closed sets yields a lattice $(\Sigma, \subseteq)$ with infimum and supremum operations

$$
\begin{aligned}
& S \sqcap T=S \cap T, \\
& S \sqcup T=\sigma(S \cup T) .
\end{aligned}
$$

Dilworth has shown that any finite lattice can be embedded into the lattice of closed sets of some classical matroid $\tilde{\mathcal{M}}$ on $E$ (relative to the family of all subsets of $E$ ) (see [2]). We study Dilworth's construction from the point of view of independence.

We define the Dilworth completion $\tilde{\rho}$ of $\rho: \mathcal{H} \rightarrow \mathbb{N}$ for all subsets $X \subseteq E$ via

$$
\tilde{\rho}(X)=\min _{H \in \mathcal{H}}\{\rho(H)+|X \backslash H|\} .
$$


It is easy to see that $\tilde{\rho}$ is normalized and unit-increasing on the collection of all subsets of $E$. Moreover, $\tilde{\rho}$ extends $\rho$ in the sense

$$
\tilde{\rho}(H)=\rho(H) \quad \text { for all } H \in \mathcal{H} .
$$

Letting

$$
\tilde{\mathcal{F}}=\{X \subseteq E|\tilde{\rho}(X)=| X \mid\}
$$

we observe

Lemma 6.1 $\tilde{\mathcal{F}}=\{X \subseteq E|| X \cap H \mid \leq \rho(H)$ for all $H \in \mathcal{H}\}$.

Proof. Note that $\tilde{\rho}(X) \leq|X|$ is always true.

If $\tilde{\rho}(X)=|X|$, then $|X \cap H| \leq \rho(H)$ follows from the definition of $\tilde{\rho}$. Conversely, if $\tilde{\rho}(X) \leq|X|-1$ holds, there is some $H \in \mathcal{H}$ with

$$
|X|-1 \geq \tilde{\rho}(X)=\rho(H)+|X \backslash H|
$$

and hence $|X \cap H| \geq \rho(H)+1$.

Lemma 6.1 shows that $\tilde{\mathcal{F}}$ is an independence system (with respect to $2^{E}$ ) and contains the $\mathcal{H}$-independence system $\mathcal{F}$.

Lemma 6.2 Assume that $\rho$ is submodular on $\mathcal{H}$. Then one has for all $X, Y \subseteq E$,

$$
\tilde{\rho}(X \cap Y)+\tilde{\rho}(X \cup Y) \leq \tilde{\rho}(X)+\tilde{\rho}(Y) .
$$

Proof. Let $S, T \in \mathcal{H}$ be such that

$$
\tilde{\rho}(X)=\rho(S)+|X \backslash S| \text { and } \tilde{\rho}(Y)=\rho(T)+|Y \backslash T| .
$$

Then we find

$$
\begin{aligned}
\tilde{\rho}(X)+\tilde{\rho}(X)= & \rho(S)+\rho(T)+|X \backslash S|+|Y \backslash T| \\
\geq & \rho(S \wedge T)+|(X \cap Y) \backslash(S \wedge T)| \\
& +\rho(S \vee T)+|(X \cup Y) \backslash(S \vee T)| \\
\geq & \tilde{\rho}(X \cap Y)+\tilde{\rho}(Y \cup Y) .
\end{aligned}
$$

Under the conditions of Lemma 6.2, $\tilde{\rho}$ is the rank function of a (classical) matroid $\tilde{\mathcal{M}}$, which we call the Dilworth completion of $\mathcal{M}=(E, \mathcal{F})$. In view of the equality (7), the greedy algorithm relative to the $\mathcal{H}$-matroid $\mathcal{M}$ may be interpreted as a special case of the greedy algorithm relative to its Dilworth completion $\tilde{\mathcal{M}}$. 
Example 6.2 With the notation of Example 2.1 and $K_{S}=\bigcup_{i \in S} K_{i}$, assume that the function $f$ is submodular on the subsets of $N$. Then

$$
\tilde{f}(X)=\min _{S \subseteq N}\left\{f(S)+\left|X \backslash K_{S}\right|\right\} \quad(X \subseteq E)
$$

is the rank function of a matroid $\tilde{\mathcal{M}}_{f}$ on $E . \tilde{\mathcal{M}}_{f}$ is the Dilworth completion of $\mathcal{F}=\{\operatorname{id}(x) \mid \mathbf{x} \in \mathbb{P}(f)\}$ with respect to the family $\mathcal{H}=\left\{K_{S} \mid S \subseteq N\right\}$. The rank function $\rho_{f}$ induced by $\mathcal{F}$ coincides with the rank function $\tilde{\rho}_{f}$ on the sets $\operatorname{id}(\mathbf{x}) \in \mathcal{D}$. So the greedy algorithm relative to $\mathcal{F}$ is a special case of the greedy algorithm relative to $\tilde{\mathcal{M}}_{f}$. Moreover, if $f$ is monotone increasing, one has

$$
\rho_{f}\left(K_{S}\right)=\tilde{\rho}_{f}\left(K_{S}\right)=f(S) \quad \text { for all } S \subseteq N,
$$

which yields the polymatroid greedy algorithm of Edmonds [6].

\section{References}

[1] M. Barnabei, G. Nicoletti, L. Pezzoli: Matroids on partially ordered sets. Adv. in Appl. Math. 21 (1998), 78-112.

[2] P. Crawley, R.P Dilworth: Algebraic Theory of Lattices. Prentice-Hall, Englewood Cliffs, 1973.

[3] B.L. Dietrich and A.J. Hoffman: On greedy algorithms, partially ordered sets, and submodular functions. IBM J. Res. \& Dev. 47 (2003), $25-30$.

[4] F.D.J. Dunstan, A.W. Ingleton, D.J.A. Welsh: Supermatroids, in: Proceedings of the Conference on Combinatorial Mathematics, Mathematical Institute Oxford, 1972, Combinatorics (1972), 72-122.

[5] P. Edelman, R.E. Jamison: The theory of convex geometries. Geom. Dedicata 19 (1985), 247-270.

[6] J. Edmonds: Submodular functions, matroids, and certain polyhedra. In: Combinatorial Structures and Their Applications, R. Guy et al. eds., Gordon and Breach, New York, 1970, 69-87.

[7] J. Edmonds: Matroids and the greedy algorithm. Math. Programming 1 (1971), 127-136.

[8] U. Faigle: The greedy algorithm for partially ordered sets. Discrete Math. 28 (1979), 153-159.

[9] U. Faigle: Geometries on partially ordered sets. J. Combin. Theory B 28 (1980), 26-51. 
[10] U. Faigle: On supermatroids with submodular rank functions. In: Algebraic Methods in Graph Theory I, Colloq. Math. Soc. J. Bolyai 25 (1981), 149-158.

[11] U. Faigle: Matroids in Combinatorial Optimization. In: Combinatorial Geometries (N. White, ed.), Encyclopedia of Math. and Its Applications 29, Cambridge University Press, New York, 1987, 161-210.

[12] U. Faigle and W. Kern: Submodular linear programs on forests. Math. Programming 72 (1996), 195-206.

[13] U. Faigle and W. Kern: On the core of ordered submodular cost games. Math. Programming 87 (2000), 483-489.

[14] U. Faigle and W. Kern: An order-theoretic framework for the greedy algorithm with applications to the core and Weber set of cooperative games. Order 17 (2000), 353-375.

[15] S. Fujishige: Dual greedy polyhedra, choice functions, and abstract convex geometries. Discrete Optimization 1 (2004), 41-49.

[16] S. Fujishige: Submodular Functions and Optimization, 2nd ed., Annals of Discrete Mathematics 58, 2005.

[17] S. Fujishige, G.A. Koshevoy, Y. Sano: Matroids on convex geometries (cg-matroids). Discrete Mathematics (to appear).

[18] D. Gale: Optimal assignments in an ordered set: an application of matroid theory. Journal of Combinatorial Theory 4 (1968) 176-180.

[19] A.J. Hoffman: On simple linear programming problems, in: Convexity: Proceedings of the Seventh Symposium in Pure Mathematics, V. Klee ed., Amer. Math. Soc., 1963, 317-327.

[20] B. Korte, L. Lovász, R. Schrader: Greedoids. Algorithms and Combinatorics 4, Springer, Heidelberg, 1991.

[21] U. Krüger: Structural aspects of ordered polymatroids. Discr. Appl. Math. 99 (2000), 125-148.

[22] G. Monge. Déblai et Remblai. Mem. de l'Académie des Science, 1781.

[23] M. Queyranne, F. Spieksma, and F. Tardella: A general class of greedily solvable linear programs. Math. Oper. Res. 23 (1998), 892-908.

[24] Y. Sano: Rank functions of strict cg-matroids. RIMS Preprint No. 1560, Research Institute for Mathematical Sciences, Kyoto University, July 2006. 
[25] Y. Sano: The greedy algorithm for strict cg-matroids. RIMS Preprint No. 1581, Research Institute for Mathematical Sciences, Kyoto University, February 2007.

[26] V.V. Shenmaier: A greedy algorithm for some classes of integer programs. Discrete Appl. Math. 133 (2004), 93-101.

[27] E. Tardos: An intersection theorem for supermatroids. J. Combin. Theory B 50 (1990), 150-159.

[28] D.J.A. Welsh: Matroid Theory, Academic Press, London, 1976. 